\title{
Microstructure and dielectric properties of BF-PFN ceramics with negative dielectric loss
}

\author{
Joanna A. Bartkowska ${ }^{1}$ (D) . Dariusz Bochenek ${ }^{1}$
}

Received: 23 April 2018 / Accepted: 3 August 2018 / Published online: 7 August 2018

(c) The Author(s) 2018

\begin{abstract}
Multiferroics with negative value of dielectric constant are very promising materials because of their modern applicability. These materials can be used as materials for the construction of electromagnetic radiation shields. The subject of the research is multiferroic $\mathrm{BiFeO}_{3}-\mathrm{PbFe}_{1 / 2} \mathrm{Nb}_{1 / 2} \mathrm{O}_{3}$ (BF-PFN) ceramics. For all multiferroic materials the following studies are conducted: SEM, EDS and the temperature dependence of dielectric constant $\varepsilon^{\prime}(T)$. Above a certain temperature (different for different chemical compositions) the value of dielectric constant reaches negative values. Such the behavior of the dielectric constant may indicate that the polarization inside the material has a reverse direction to the external electric field. That is, the electric field inside the material counteracts the applied external electric field. The obtaining materials also show negative dielectric losses. The Axelrod model is used to explain the mechanism that causes negative dielectric loss.
\end{abstract}

\section{Introduction}

Multiferroics are materials that are characterized by at least two ferroic features that occur in same phase. In recent years, magnetoelectric materials have become a frequently studied multiferroic materials [1-6]. In magnetoelectric multiferroic materials, polarization and magnetization occur in the same phase. Due to the magnetoelectric effect, which occurs in these materials, magnetization and polarization are coupled together $[7,8]$. From the point of view of potential applications in electronics and spintronics, magnetoelectric materials seem to be the most promising, because they may find application as sensors, transducers, microwave devices, read/ write devices [9-14].

Among the magnetoelectric multiferroics, an interesting group of materials are materials whose dielectric constant is negative. Dolgov et al. [15] showed that the existence of systems (materials) with negative dielectric constant is possible and this is in line with the principles of the electrodynamics. Such materials may exist and are stable [15]. At the moment, there are only a few papers that present the results

Joanna A. Bartkowska

joanna.bartkowska@us.edu.pl

1 Faculty of Computer Science and Materials Science, Institute of Technology and Mechatronics, University of Silesia in Katowice, 12, Żytnia St., 41-200 Sosnowiec, Poland of testing materials with a negative value of dielectric constant [16-21]. The mechanism that causes the negative value of the dielectric constant has not been fully understood and explained yet. Materials with a negative value of dielectric constant may also have negative dielectric loss. The phenomenon of negative dielectric loss has been found, among others in sol-gel glasses. Axelrod et al. [22] have proposed a model of mechanism that causes negative dielectric loss. The results of research of materials with negative dielectric constant and/or negative dielectric loss are beginning to appear in the literature. Materials with such physical properties may be the starting points for obtaining materials with the so-called Negative Index Material [23-26].

The purpose of this work was to obtain $\mathrm{BiFeO}_{3}-\mathrm{PbFe}_{1 / 2} \mathrm{Nb}_{1 / 2} \mathrm{O}_{3}(\mathrm{BF}-\mathrm{PFN}$ ) materials (ceramic solid solutions) with different $\mathrm{BiFeO}_{3}(\mathrm{BF})$ content in relation to $\mathrm{PbFe}_{1 / 2} \mathrm{Nb}_{1 / 2} \mathrm{O}_{3}$ (PFN) and to study the dielectric properties of the obtained samples. Both PFN [27-31] and $\mathrm{BF}$ [32-34] materials belong to the group of multiferroic materials. The paper presents the results of research of morphology of fracture, qualitative and quantitative analysis of chemical elements contained in the obtained samples. The results of temperature dielectric tests were also presented, which confirmed that the BF-PFN material is characterized by interesting dielectric properties, which allows to include this material into the group of materials with a Negative Index Material. 


\section{Experimental}

The object of investigations was multiferroic $\mathrm{BiFeO}_{3}-\mathrm{PbFe}_{1 / 2} \mathrm{Nb}_{1 / 2} \mathrm{O}_{3}$ ceramic solid solutions (BF-PFN) with different content of $\mathrm{BiFeO}_{3}(\mathrm{BF})$ component in relation to $\mathrm{PbFe}_{1 / 2} \mathrm{Nb}_{1 / 2} \mathrm{O}_{3}$ (PFN) component $[35,36]$. Three multiferroic ceramics obtained from simple oxides with percentages contain BF/PFN: 60/40 (BF60-PFN40), 70/30 (BF70-PFN30), 80/20 (BF80-PFN20), respectively were tested. Solid solutions of the BF-PFN were obtained from simple oxides: $\mathrm{PbO}(99.9 \%, \mathrm{POCH}), \mathrm{Fe}_{2} \mathrm{O}_{3}(99.9 \%, \mathrm{POCH})$, $\mathrm{Nb}_{2} \mathrm{O}_{5}$ (99.9\%, Sigma-Aldrich) and $\mathrm{Bi}_{2} \mathrm{O}_{3}$ (99.9\%, Aldrich). The powders were weighed in a suitable, stoichiometric manner, while the lead oxide $\mathrm{PbO}$ was weighed with excess of $3.5 \mathrm{wt} \%$ (to compensate lead evaporation during sintering). Then the ceramic powders were mixed and milled in a planetary ball mill for $15 \mathrm{~h}$ and next were successively dried and calcined at $1173 \mathrm{~K} / 2 \mathrm{~h}$. The obtained powders were uniaxially pressed into discs with a diameter of $10 \mathrm{~mm}$ and the thickness of about $3 \mathrm{~mm}$, using polyvinyl alcohol as a binder. Such prepared ceramic discs have been sintered in alumina crucibles at $1323 \mathrm{~K}$ for $2 \mathrm{~h}$, with the heating rate of about $2.5 \mathrm{~K} / \mathrm{min}$ (for all three compositions of the BF-PFN material). In the next step the ceramic samples were grinded, polished and annealed at $973 \mathrm{~K} / 5 \mathrm{~min}$. to remove mechanical stresses that may have occurred during mechanical machining. For the electrical tests, silver electrodes (P-120 supplier Polish State Mint) were applied on both surfaces of the ceramic samples (burning method).

X-ray powder-diffraction data (XRD) of the BF-PFN ceramic materials were carried out at room temperature $(R T)$ on a diffractometer Phillips X'Pert APD $(\mathrm{Cu}-\mathrm{K} \alpha$ radiation). The data were collected in the $2 \theta$ range from $10^{\circ}$ to $65^{\circ}$ in steps of 0.02 degrees with an integration time of $4 \mathrm{~s} / \mathrm{step}$. Microscopic investigations were performed on the fracture of samples (after breaking the samples), using the scanning electron microscope (JEOL JSM-7100F TTL LV). The chemical composition analysis in microareas was performed by the standard method, using an energy dispersive spectrometer (EDS). The SEM/EDS studies were performed by the accelerating voltage $15 \mathrm{kV}$, and with $\mathrm{Au}$ sputtering on the surface of samples, using Vacuum Evaporator JEE-4X (JEOL). Au sputtering on the sample was the last stage of sample preparation for imaging and analysis with the electron microscope. Dielectric constant and loss tangent were measured on the $1 \mathrm{~V}$ amplitude of the driving signal using LCR meter (QuadTech 1920 Precision) at temperature range from $R T$ to $923 \mathrm{~K}$, for several frequencies of measurement field (range from $20 \mathrm{~Hz}$ to $1 \mathrm{MHz}$ ). DC electrical conductivity has been measured using a Keithley 6517B electrometer (high resistance meter) in temperature range from $R T$ to $570 \mathrm{~K}$.

\section{Results and discussion}

The XRD test of the multiferroic BF-PFN ceramic powders after sintering have been presented in Fig. 1. On diffraction patterns for all BF-PFN samples are visible the lines connected with the $\mathrm{BiFeO}_{3}$ and $\mathrm{PbFe}_{1 / 2} \mathrm{Nb}_{1 / 2} \mathrm{O}_{3}$ materials as well as the lines connected with of foreign phase $\left(\mathrm{Bi}_{25} \mathrm{FeO}_{40}\right)$. A secondary undesirable phase $\mathrm{Bi}_{25} \mathrm{FeO}_{40}$ (JCPDS card no. 01-073-9538) is created during the technological process. The largest amount of foreign phase occurs for the BF80-PFN20 sample. In the case of the $\mathrm{BiFeO}_{3}(\mathrm{BF})$ component the best fit to the measured data was obtained with the pattern JCPDS card no. 86-1518 (a polycrystalline rhombohedral distorted perovskite structure with a space group of $\mathrm{R} 3 c$ ). In the case of the $\mathrm{PbFe}_{1 / 2} \mathrm{Nb}_{1 / 2} \mathrm{O}_{3}$ (PFN) component the best fit to the measured data was obtained with the pattern JCPDS card no. 04-009-5124 (a perovskite structure with the tetragonal symmetry and $\mathrm{P} 4 \mathrm{~mm}$ space group).

Results of morphology studies of breakthrough of the samples have been presented in Fig. 2. Microstructures of BF60-PFN40 and BF70-PFN30 ceramics are characterized by well-shaped grins grains (with clearly visible grain boundaries and with sharp edges of the grains) of different size (Fig. 2a, b, respectively). The microstructure of multiferroic BF80-PFN20 sample (Fig. 2c) is characterized by worse shaped grains, compared to microstructure of BF60-PFN40 and BF70-PFN30 ceramic materials. The microstructure of BF60-PFN40 and BF70-PFN30 multiferroic ceramics reveals clear grains boundaries after breaking the samples. In the case of the BF60-PFN40 ceramic material only one type of fractures appears after breaking the samples, namely along the grain boundaries (Fig. 2a). In the case of the BF70-PFN30 ceramics two types of fracture appears, the first one (main) along the grain boundaries, but also single fractures through grains are present in

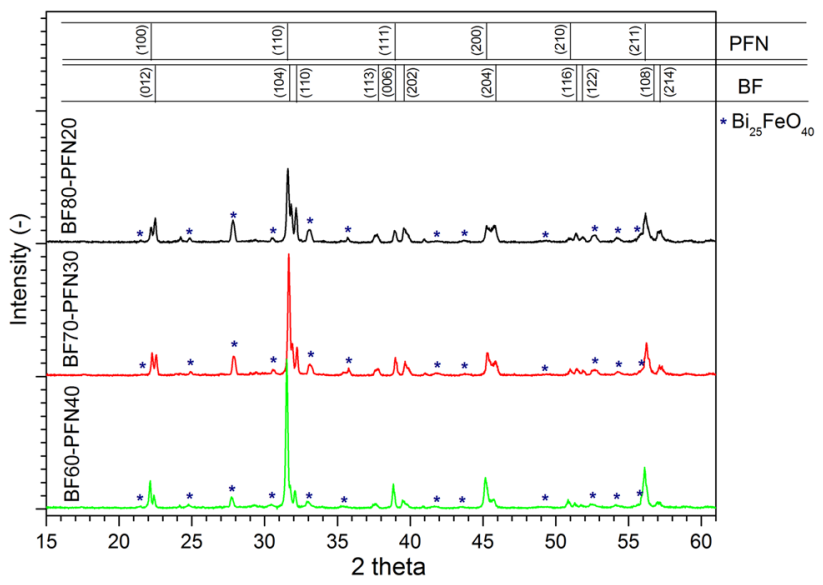

Fig. 1 The X-ray spectra of the multiferroic BF-PFN powders 

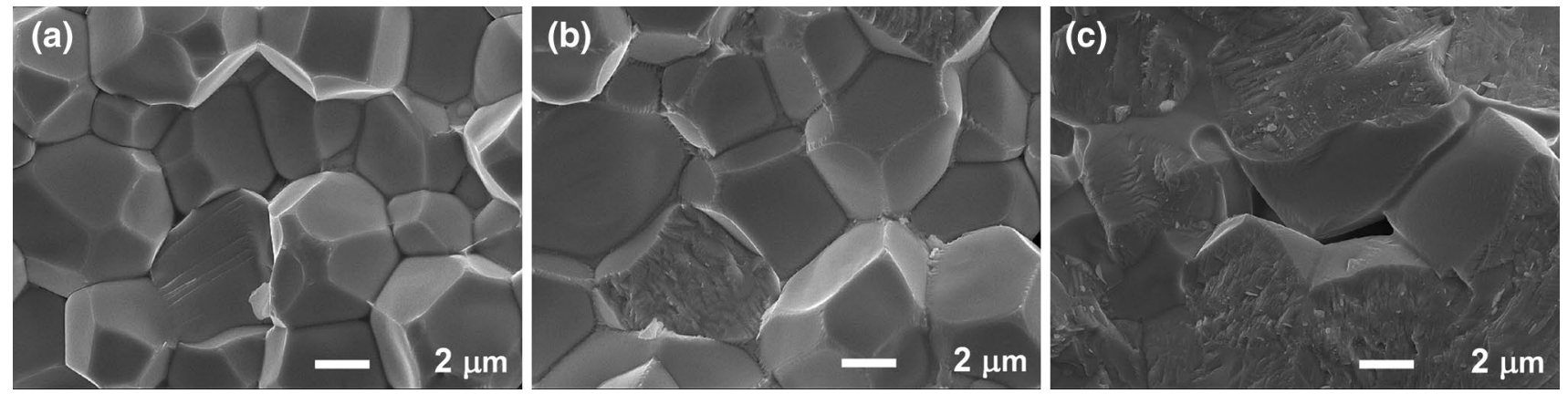

Fig. 2 SEM images of the microstructure of fracture of magnetoelectric BF-PFN-type material, BF60-PFN40 (a), BF70-PFN30 (b), BF80PFN20 (c) samples, respectively

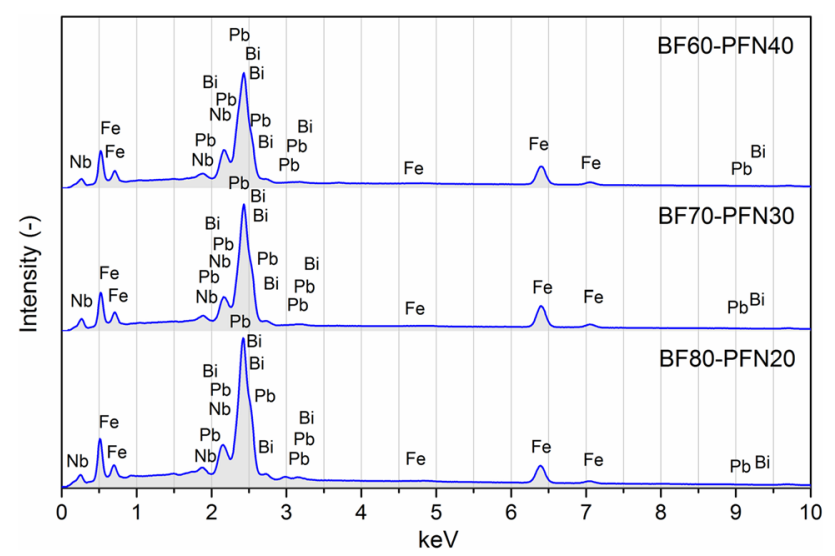

Fig. 3 The EDS analysis of chemical elements of BF60-PFN40, BF70-PFN30, BF80-PFN20 samples, respectively

this material (Fig. 2b). The type of cracks along the grain boundaries is becoming dominating in this ceramic sample. A mixed nature of fracture occurs in BF80-PFN20 material, that is, fractures occur along boundaries as well as through grains (Fig. 2c).

An EDS analysis of investigated multiferroic ceramic samples qualitatively confirmed the assumed share of the specific components and it showed that the materials do not contain any impurities (Fig. 3). The results of the percentage of individual components of the BF-PFN ceramic samples are the averaging of 10 randomly-chosen areas from the surface of the sample. The tested materials showed quite stable chemical composition. The $\mathrm{Nb}_{2} \mathrm{O}_{5}$ and $\mathrm{BiO}_{2}$ are the most stable behavior in both BF60-PFN40 and BF80-PFN20 ceramic samples, while $\mathrm{Fe}_{2} \mathrm{O}_{3}$ and $\mathrm{BiO}_{2}$ are the most stable behavior in BF70-PFN30 ceramic sample (Table 1).

The analysis of the elements distribution showed some differences in the chemical composition but all deviations between the theoretical content of chemical elements in the tested materials and their real content are within the acceptable range.

The temperature dependencies of dielectric constant $\varepsilon^{\prime}$ for the BF60-PFN40 ceramic sample is shown in Fig. 4a. The dielectric constant increases with increasing temperature up to $643 \mathrm{~K}$, and then decreases with further growth of temperature. The dielectric constant reaches a value of zero at a temperature of $743 \mathrm{~K}$, for all frequencies of the measuring electric field. A further increase in temperature causes the dielectric constant value to drop below zero. The dielectric constant takes negative values. The location of the maximum of the dielectric constant does not depend on the frequency of the measuring electric field and it may correspond to the ferroelectric-paraelectric phase transition. The second maximum of dielectric constant for frequencies in the range from 20 to $500 \mathrm{kHz}$ appears below the phase transition temperature (the inset of Fig. 4a). The location of this maximum depends on the frequency of measuring field, i.e. the relaxation processes are responsible for its origin. The part of $\mathrm{Fe}^{3+}$ ions which present in the material could be reduced to $\mathrm{Fe}^{2+}$ ions during the sintering process. The potential barrier between these two types of ions affects the movement of charges, causing the relaxation process to appear in tested BF-PFN ceramic material [37].
Table 1 Theoretical and experimental percentage of chemical elements in all studied BF-PFN-type samples

\begin{tabular}{|c|c|c|c|c|c|c|}
\hline & \multicolumn{2}{|c|}{ BF60-PFN40 } & \multicolumn{2}{|c|}{ BF70-PFN30 } & \multicolumn{2}{|c|}{ BF80-PFN20 } \\
\hline & Theor. $(\%)$ & Exper. (\%) & Theor. (\%) & Exper. (\%) & Theor. (\%) & Exper. (\%) \\
\hline $\mathrm{PbO}$ & 27.94 & 29.45 & 21.07 & 22.53 & 14.12 & 15.77 \\
\hline $\mathrm{Fe}_{2} \mathrm{O}_{3}$ & 19.99 & 18.90 & 21.35 & 21.27 & 22.73 & 21.54 \\
\hline $\mathrm{Nb}_{2} \mathrm{O}_{5}$ & 8.32 & 8.82 & 6.27 & 6.56 & 4.20 & 4.77 \\
\hline $\mathrm{BiO}_{2}$ & 43.75 & 42.83 & 51.31 & 49.64 & 58.95 & 57.92 \\
\hline
\end{tabular}



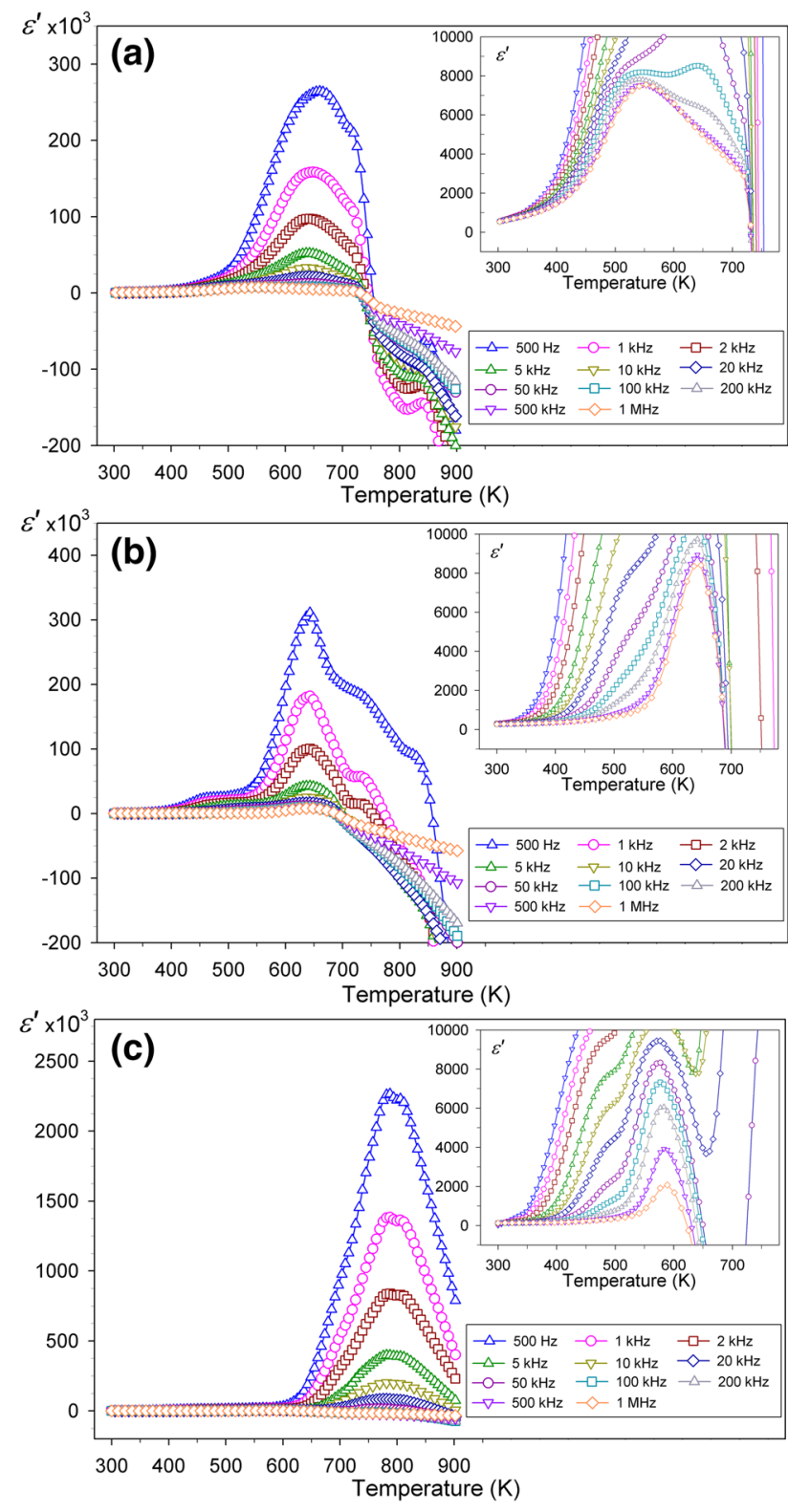

Fig. 4 Temperature dependencies of dielectric constant $\varepsilon^{\prime}$ for BF60PFN40 (a), BF70-PFN30 (b), BF80-PFN20 (c) samples, respectively

Figure $4 \mathrm{~b}$ shows the temperature dependencies of dielectric constant $\varepsilon^{\prime}$ for BF70-PFN40 ceramic sample. The dielectric constant increases with increasing temperature similar to dielectric constant for BF60-PFN40 sample. The maximum of dielectric constant appears at $628 \mathrm{~K}$ for all frequencies of the measuring electric field. This maximum is independent of frequencies of measuring field. The ferroelectric-paraelectric phase transition may be responsible for its appearance. A further increase in temperature causes the dielectric constant to decrease to negative values. The second maximum of dielectric constant, for frequencies in the range from $500 \mathrm{~Hz}$ to $20 \mathrm{kHz}$ appears below the phase transition temperature similar to the BF60-PFN40 material (the inset of Fig. 4b). The reason for the creation of this maximum, are the relaxation processes that take place in the tested material.

The temperature dependencies of dielectric constant $\varepsilon^{\prime}$ for BF80-PFN20 ceramic material is shown in Fig. 4c. The values of dielectric constant increase with increasing temperature and reach the maximum value at $793 \mathrm{~K}$. The clear maximum of dielectric constant occurs in the same temperature for all measuring frequencies. Therefore the temperature of this maximum corresponds to the ferroelectric-paraelectric phase transition in BF80-PFN20 material. A further increase in temperature causes decreasing of the dielectric constant. The values of the dielectric constant, in the tested temperature range, decrease up to negative values for the frequency of measuring electric field in the range from 20 to $500 \mathrm{kHz}$, while the value of dielectric constant remains positive for remaining frequencies (i.e. from $500 \mathrm{~Hz}$ to $10 \mathrm{kHz}$ ).

The inset of Fig. 4c shows the dielectric anomaly at temperatures below phase transition temperature (the dielectric anomaly appears at the range from 433 to $583 \mathrm{~K}$ ). The critical temperature increases with increasing frequency of measuring electric field. The position change of this maximum from the temperature (at different measuring frequencies), indicates the occurrence of dielectric relaxation in the material under study. This relaxation behavior may come from the properties of the $\mathrm{BF}$ component of the final BF-PFN solid solution [38].

Negative values of dielectric constant, which clearly appear, in the tested temperature range, for both BF60-PFN40 and BF70-PFN30 materials, may indicate a change in the electrical character of these materials. Namely the electrical character of tested materials is changing from capacitive to inductive one [39]. It can be assumed, by analogy with a diamagnetics in the magnetic field that the material with negative dielectric constant has dia-electric properties [40, 41], i.e. the electric field inside the material has the opposite direction to the applied external electric field.

The value of temperature of ferroelectric-paraelectric phase transition is different for various chemical compositions of the BF-PFN ceramics (Fig. 4a-c). The lowest phase transition temperature $(628 \mathrm{~K})$ reveals the BF70-PFN30 material, while the highest $(793 \mathrm{~K})$ reveals the BF80-PFN20 sample. The BF60-PFN40 sample is characterized by a phase transition temperature equal to $643 \mathrm{~K}$. The results of these studies show that the percentage of BF and PFN components in ceramics affects the value of the phase transition temperature. Increasing the amount of BF (only to a certain value) in relation to PFN affects the reduction of the phase transition temperature. There is a limit ratio of the content of $\mathrm{BF}$ and PFN in ceramics above which the temperature of the phase transition increases. The dependence of the phase transition temperature on the percentage of the BF and PFN 
components, in tested ceramic materials, can be related to influence the BF and PFN components on the anchored of the ferroelectric domain walls. In the BF70-PFN30 ceramics, the ferroelectric domain walls are the least anchored. The least energy is required to release them therefore the phase transition temperature is the lowest among the materials tested. While in the BF80-PFN20 material, the ferroelectric domain walls are most strongly anchored. In order to free the domain walls, the largest energy, in relation to the other studied materials, is required. The confirmation of this fact may be the highest temperature of ferroelectric - paraelectric phase transition (in relation to the other materials tested).

Temperature dependencies of dielectric loss tangent for all tested materials are presented in Fig. 5. Comparing the results for all BF-PFN ceramic samples may be observed that the negative loss phenomenon takes place at different temperature regions, as shown in Fig. 5.

Figure 5a shows that dielectric loss tangent for BF60-PFN40 sample increases with increasing temperature. The first maximum of dielectric loss appears at $623 \mathrm{~K}$ (the inset of Fig. 5a), the second one occurs at 723 K. Next, dielectric loss decrease with further increasing temperature and at above $743 \mathrm{~K}$ they reach negative values, for all frequencies of the measuring field.

Temperature dependencies of dielectric loss tangent for BF70-PFN30 sample are presented in Fig. 5b. The value of dielectric loss tangent increases with the temperature rise to a certain value, and next with further increasing temperature the value of the $\tan \delta$ begins to decrease up to the negative values. A small maximum appears on the temperature dependencies of dielectric loss tangent at $523 \mathrm{~K}$ (the inset of Fig. 5b). This small maximum of dielectric loss is related with the relaxation processes taking place in the tested BF-PFN material. Further increasing temperature causes decreasing of the values of dielectric loss tangent up to negative values. Negative values of the $\tan \delta$ occur at different temperatures for different frequencies of measuring field (Fig. 5c).

Temperature dependencies of dielectric loss tangent for BF80-PFN20 sample were presented in Fig. 5c. There is only one maximum on temperature dependencies of dielectric loss tangent for BF80-PFN20 material. The values of the $\tan \delta$ increasing with the temperature and reach a maximum in the temperature range from 583 to $653 \mathrm{~K}$ (depending on the frequency of the measuring field). Next, with further increasing temperature, the values of the $\tan \delta$ begin to decreases. Dielectric loss reach negative values, in the studied temperature range, only for several frequencies of measuring field (Fig. 5c).

Positive dielectric loss corresponds to the normal relaxation processes that are associated with process of absorption and emission of energy. Axelrod et al. showed in their
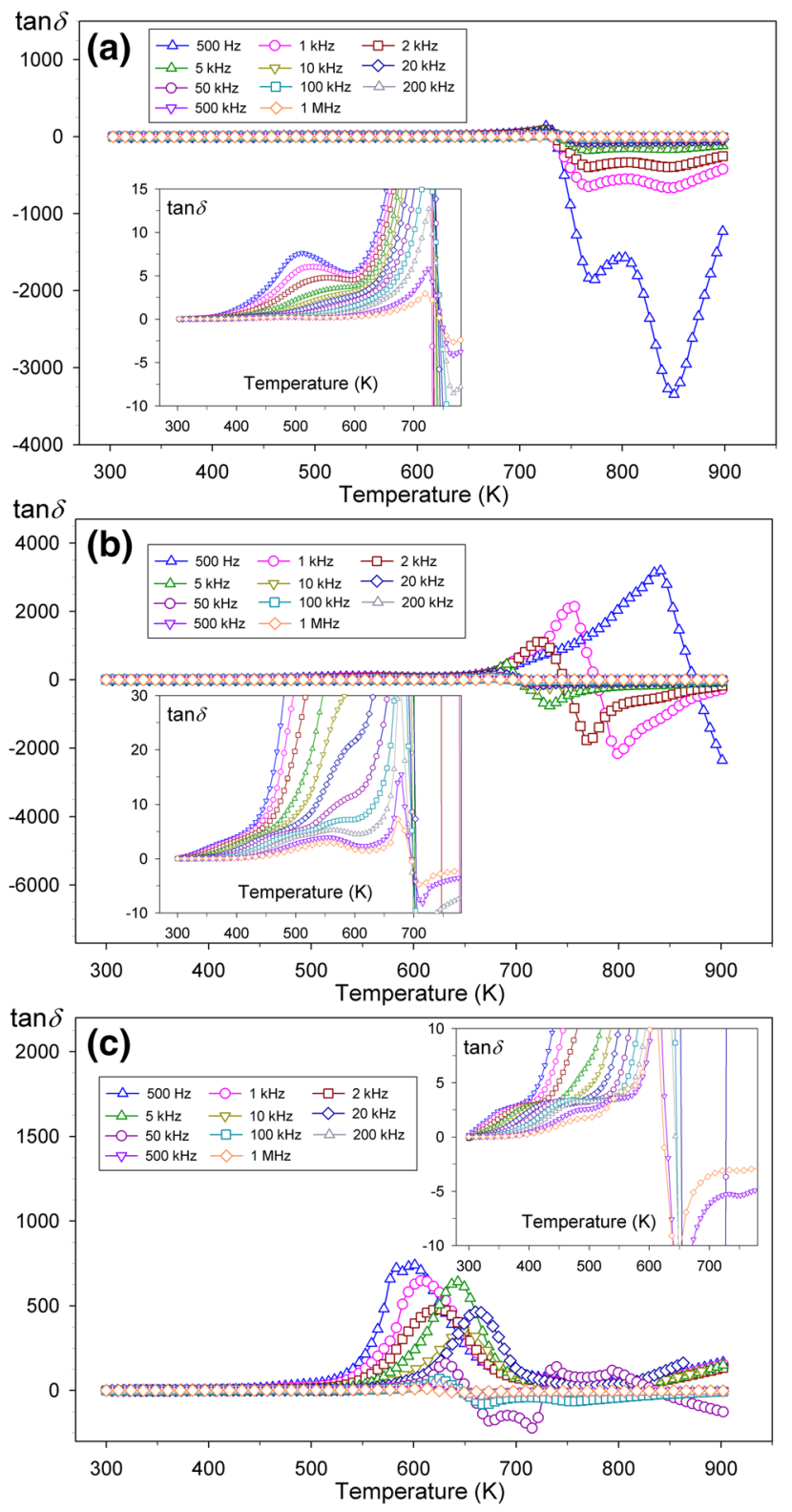

Fig. 5 Temperature dependencies of dielectric loss tangent $\tan \delta$ for BF60-PFN40 (a), BF70-PFN30 (b), BF80-PFN20 (c) samples, respectively

work [22] that a negative loss phenomenon is related to the fact that more energy must be released than absorbed. According to, the principle of energy conservation, the total energy in the ceramic sample must be conserved. Therefore, there must be some mechanism in this material that allows you to store energy and release it only at certain temperatures and at certain frequencies. The negative loss phenomenon may be attributed to local noncompensated charges, which may accumulate inside the real porous material on the interface of the pores. These charges may be anchored via non-bonding orbitals, full 
or empty for positive or negative ions, respectively. This assumption allows explain the mechanism of separation of the space charges. The separation of charge leads to the accumulation of energy and this is a metastable state. This metastable state may be eliminated under certain conditions such us temperature and frequency. The specified temperature and frequency may cause avalanche recombination of charges, which results in the effect of energy emission [22].

Figure 6 shows the Arrhenius plot i.e. the dependence of the logarithm of the maximum value of dielectric constant (for the relaxation maximum) against the inverse of the temperature. The Arrhenius plot was used to analyze relaxation processes. To analyze the dielectric relaxation process, the activation energy has been calculated for tested BF-PFN ceramic samples. The activation energy was calculated by fitting the Arrhenius law [42] to the results shown in Fig. 6. The activation energies, which were determined from straight fitting lines, are equal: $1.18 \mathrm{eV}, 1.29 \mathrm{eV}$ and $1.38 \mathrm{eV}$, for BF60-PFN40, BF70-PFN30 and BF80-PFN20 ceramic samples, respectively. Such values of activation energy correspond to the relaxation process, which may be related to the occurrence of ionization of oxygen vacancies [43].

Temperature measurements of the DC electrical conductivity of the multiferroic BF-PFN ceramic samples are show in Fig. 7. At lower temperatures, the lowest electrical conductivity has BF60-PFN40 sample, while the highest electrical conductivity has BF80-PFN20 sample. At higher temperatures, the temperature plots have a similar shape for all BF-PFN ceramic samples.

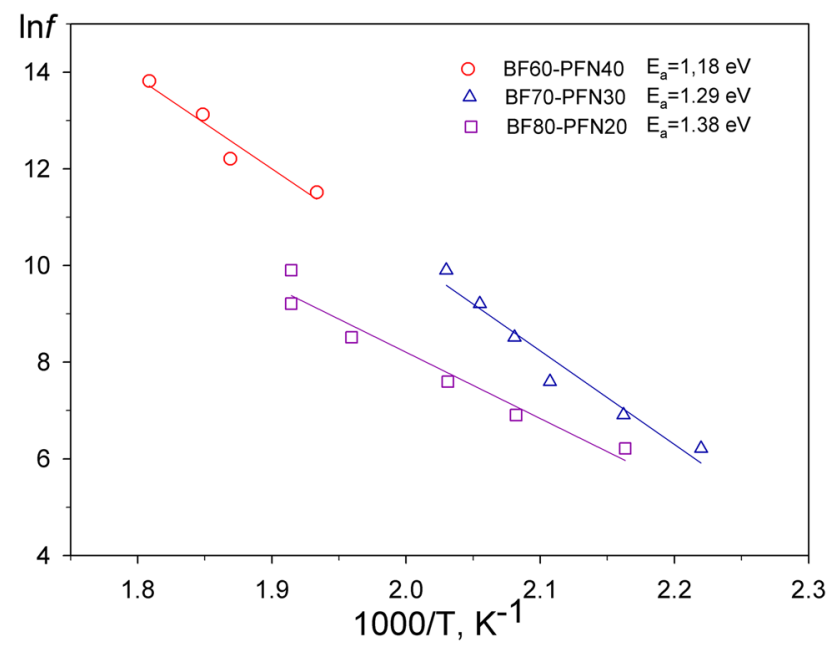

Fig. 6 The Arrhenius plot for BF60-PFN40, BF70-PFN30 and BF80-PFN20 ceramic samples, the solid lines are fitting data

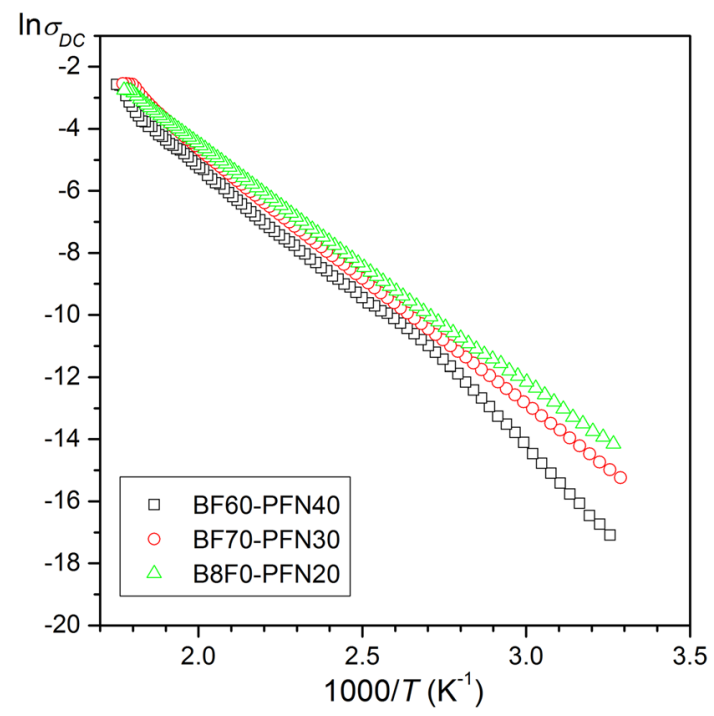

Fig. 7 The $\ln \sigma_{\mathrm{DC}}(1000 / T)$ relationship for the BF-PFN ceramic samples

\section{Conclusion}

The $\mathrm{BiFeO}_{3}-\mathrm{PbFe}_{1 / 2} \mathrm{Nb}_{1 / 2} \mathrm{O}_{3}$ ceramic solid solutions (BF-PFN) were prepared from simple oxides, using a conventional solid state technique. The morphology of fractures and dielectric properties were investigated.

The first dielectric maxima on the $\varepsilon^{\prime}(T)$ graphs, which are connected with dielectric relaxation, appear at high frequencies for BF60-PFN40 and BF70-PFN30 ceramic samples and it appears at low frequencies for BF80-PFN20 sample. The activation energy for the BF-PFN ceramic materials is in the range from 1.18 to $1.38 \mathrm{eV}$.

The negative dielectric constant was observed at a broad range of frequencies namely below $500 \mathrm{~Hz}$ and up to $1 \mathrm{MHz}$ for both BF60-PFN40 and BF70-PFN30 ceramic samples. The decrease in the value of the dielectric constant below zero may be related to the change of the electrical nature of the material from capacitive to inductive. Negative values of dielectric loss were explained based on the Axelrod model, which associates dielectric loss with energy emissions. Negative dielectric loss therefore indicates that more energy has been emitted than absorbed. Increase energy emission is associated with the process of avalanche release of localized charges in some of the ceramic materials. This rare phenomenon may occur in certain temperature ranges as well as for specific frequencies.

Open Access This article is distributed under the terms of the Creative Commons Attribution 4.0 International License (http://creat ivecommons.org/licenses/by/4.0/), which permits unrestricted use, 
distribution, and reproduction in any medium, provided you give appropriate credit to the original author(s) and the source, provide a link to the Creative Commons license, and indicate if changes were made.

\section{References}

1. P. Kumar, Integr. Ferroelectr. 131, 25 (2011)

2. W. Eerenstein, N.D. Mathur, J.F. Scott, Nat. Mater. 442, 759 (2006)

3. W. Kleemann, P. Borisov, V.V. Shvartsman, S. Bedanta, EPJ Web Conf. 29, 00046 (2012)

4. N.A. Spaldin, MRS Bull. 42, 385 (2017)

5. M. Fiebig, T. Lottermoser, D. Meier, M. Trassi, Nat. Rev. Mater. 1, 16046 (2016)

6. M.-Ch Kao, H.-Z. Chen, S.-L. Young, K.-H. Chen, J.-L. Chiang, J.-B. Shi, Materials 10, 1327 (2017)

7. K.F. Wang, J.M. Liu, Z.F. Ren, Adv. Phys. 58, 321 (2009)

8. D. Khomskii, Physics 2, 20 (2009)

9. J.F. Scott, J. Mater. Chem. 22, 4567 (2012)

10. M.M. Vopson, Crit. Rev. Solid State 40, 223 (2015)

11. P. Němec, M. Fiebig, T. Kampfrath, A.V. Kimel, Nat. Phys. 14, $229(2018)$

12. C.-W. Nan, M.I. Bichurin, S. Dong, G. Srinivasan, J. Appl. Phys. 103, 031101 (2008)

13. D. Pandey, A.K. Singh, S. Baik, Acta Crystallogr. A 64, 192 (2008)

14. J. Ryu, S. Priya, K. Uchino, H.-E. Kim, J. Electroceram. 8, 107 (2002)

15. O.V. Dolgov, D.A. Kirzhnits, E. Maksimov, Rev. Mod. Phys. 53, $81(1981)$

16. K. Lee, A.J. Heeger, Phys. Rev. B 68, 035201 (2003)

17. S. Ameen, V. Ali, M. Zulfequar, M. Haq, M. Husain, Curr. Appl. Phys. 7, 215 (2007)

18. Q. Hou, K. Sun, P. Xie, K. Yan, R. Fan, Y. Liu, Mater. Lett. 169, $86(2016)$
19. H. Yan, C. Zhao, K. Wang, L. Deng, M. Ma, G. Xu, Appl. Phys. Lett. 102, 062904 (2013)

20. R. Liu, A. Degiron, J.J. Mock, D.R. Smith, Appl. Phys. Lett. 90, 263504 (2007)

21. D. Schurig, J.J. Mock, D.R. Smith, Appl. Phys. Lett. 88, 041109 (2006)

22. E. Axelrod, A. Puzenko, Y. Haruvy, R. Reisfeld, Y. Feldman, J. Non-Cryst. Solids 352, 4166 (2006)

23. S.M. Xiao, V.P. Drachev, A.V. Kildishev, X.J. Ni, U.K. Chettiar, H.K. Yuan, V.M. Shalaev, Nature 466, 735 (2010)

24. D.R. Smith, W.J. Padilla, D.C. Vier, S.C. Nemat-Nasser, S. Schultz, Phys. Rev. Lett. 84, 4184 (2000)

25. B. Li, G. Sui, W.H. Zhong, Adv. Mater. 21, 4176-4180 (2009)

26. W.J. Padilla, D.N. Basov, D.R. Smith, Mater. Today 9, 28 (2006)

27. O. Raymond, R. Font, J. Portelles, J.M. Siqueiros, J. Appl. Phys. 109, 094106 (2011)

28. D. Bochenek, J. Alloy. Compd. 504, 508 (2010)

29. D. Bochenek, Z. Surowiak, J. Alloy. Compd. 480, 732 (2009)

30. D. Bochenek, Z. Surowiak, J. Krok-Kowalski, J. PoltierovaVejpravova, J. Electroceram. 25, 122 (2010)

31. D. Bochenek, J. Dudek, Eur. Phys. J. 154, 19 (2008)

32. J.M. Caicedo, J.A. Zapata, M.E. Gomez, P. Prieto, J. Appl. Phys. 103, 07E306 (2008)

33. V.B. Naik, R. Mahendiran, Solid State Commun. 149, 754 (2009)

34. J.A. Bartkowska, J. Ilczuk, Int. J. Thermophys. 31, 1 (2007)

35. J.A. Bartkowska, Int. J. Thermophys. 32, 739 (2011)

36. D. Bochenek, P. Guzdek, J. Magn. Magn. Mater. 323, 369 (2011)

37. M.H. Abdullah, A.N. Yusoff, J. Mater. Sci. 32, 5817 (1997)

38. F. Huang, X. Lu,. W. Lin, W. Cai, X. Wu, Y. Kan, H. Sang, J. Zhu, Appl. Phys. Lett. 90, 252903 (2007)

39. X.Y. Mao, W. Wang, X.B. Chen, Solid State Commun. 147, 186 (2008)

40. C. Caloz, Mater. Res. Soc. Symp. Proc. 919, 0919-J02 (2006)

41. A.K. Srivastava, A.C. Pandey, R. Kripal, S.H. Lee, Soft Mater. 12, 284 (2014)

42. D. Bochenek, P. Niemiec, P. Guzdek, M. Wzorek, Mater. Chem. Phys. 195, 199 (2017)

43. P.R. Mandal, T.K. Nath, J. Alloys Compd. 628, 379 (2015) 